\title{
Corela
}

Cognition, représentation, langage

HS-29 | 2019

Questions et exclamations au prisme de plusieurs approches linguistiques

\section{Point de vue pragmatique sur l'exclamation}

\section{Laure LANSARI}

\section{(2) OpenEdition \\ Journals}

Édition électronique

URL : http://journals.openedition.org/corela/9020

DOI : $10.4000 /$ corela.9020

ISSN : 1638-573X

Éditeur

Cercle linguistique du Centre et de l'Ouest - CerLICO

Référence électronique

Laure LANSARI, « Point de vue pragmatique sur l'exclamation », Corela [En ligne], HS-29 | 2019, mis en ligne le 09 septembre 2019, consulté le 28 octobre 2019. URL : http://journals.openedition.org/corela/ 9020 ; DOI : 10.4000/corela.9020

Ce document a été généré automatiquement le 28 octobre 2019

\section{(c) (i) (3) (2)}

Corela - cognition, représentation, langage est mis à disposition selon les termes de la licence Creative Commons Attribution - Pas d'Utilisation Commerciale - Partage dans les Mêmes Conditions 4.0 International. 


\title{
Point de vue pragmatique sur l'exclamation $^{1}$
}

\author{
Laure LANSARI
}

\section{Introduction}

1 Que ce soit en français ou en anglais, le terme même "exclamation " peut prêter à confusion : « exclamation » désigne le processus, c'est-à-dire le fait de s'exclamer, mais aussi le produit stabilisé de ce processus dynamique, c'est-à-dire l'énoncé observable par le linguiste. Toute analyse linguistique de l'exclamation doit donc d'abord passer par une remise à plat de la métalangue. C'est en ce sens que R. Quirk et al. (1985) proposent de réserver le terme «exclamation » au seul processus et d'utiliser le terme " exclamative " pour désigner certaines structures de l'anglais qui semblent spécialisées dans l'exclamation : les structures en what et how qui marquent dans leur morphologie un jugement scalaire de haut degré, ce que fait apparaitre une glose mettant en jeu l'adverbe intensifieur so. Le corpus sur lequel se fonde le présent volume offre deux de ces structures : une structure exclamative dite « directe » et réduite puisqu'averbale 1. 19 " what a convenient location ", glosable par this location is so convenient!; une structure exclamative dite « indirecte » (" embedded exclamative » dans la terminologie en anglais) 1. 170, «It's just incredible, Blanche, how well you're looking », que l'on peut gloser par you're looking so well!

2 La distinction terminologique proposée par R. Quirk et al. (1985) entre exclamation et exclamative amène à opérer une disjonction fondamentale entre fonction et forme, d'autant plus nécessaire qu'il n'existe pas de rapport bi-univoque entre forme exclamative et fonction d'exclamation. Cela signifie en premier lieu que l'étude de l'exclamation comme fonction ne peut se réduire, sur le plan sémasiologique, à l'analyse des seules formes exclamatives en what/how : le linguiste doit aussi prendre en compte ce que l'on peut appeler les autres «formes d'exclamation ». Si l'on observe le corpus, on est ainsi frappé par deux éléments : la rareté des "exclamatives» (au nombre de deux, voir plus haut) d'une part, la variété morphosyntaxique des formes 
d'exclamation d'autre part. On note ainsi la présence de structures qui ne sont pas exclamatives dans la forme, mais qui appartiennent aux trois autres types de phrase traditionnellement définies dans les grammaires: les déclaratives, les interrogatives, les impératives. Les déclaratives, comme 11. 28-29 «Oh, I spy, I spy!», constituent la forme la plus courante du corpus, mais l'on trouve également une forme que l'on peut rapprocher des interrogatives par la présence de why 1.66 "Why, that you had to live in these conditions! $»^{2}$, ainsi qu'un énoncé impératif 1.15 «turn that over-light off!». On remarque également l'emploi de nombreuses formes moins prototypiques, averbales, nommées "free Noun Phrases " par Michaelis (2001: 1048) ou "nominal exclamatives" pour Rett (2011) - citons par exemple 1. 10 « Stella, oh, Stella, Stella! Stella for Star!».

Cela montre bien qu'une définition traditionnelle de l'exclamation en tant que type de phrase opposé aux déclaratives, interrogatives et impératives ne suffit pas ${ }^{3}$. Le corpus choisi apparaît en cela très représentatif de la problématique de l'exclamation et corrobore les recherches menées, dans le cadre du projet ANR Emphiline ${ }^{4}$, sur l'exclamation dans un corpus d'interaction: les formes considérées comme prototypiques par les grammaires - à savoir les exclamatives en what et how - sont quantitativement marginales. On voit ainsi se dessiner une lacune des grammaires et de certaines études linguistiques ${ }^{5}$ qui réduisent parfois l'exclamation à ces structures qui ne sont pourtant pas les plus fréquentes.

4 L'autre conséquence, moins évidente et moins notée dans la littérature, de la disjonction fondamentale à opérer entre fonction d'exclamation et forme exclamative est que les structures exclamatives sont susceptibles d'être utilisées sans qu'il y ait une véritable exclamation de la part du locuteur. Ainsi, Celle \& Lansari (2015) notent à propos de certaines exclamatives en "what a + noun " (notamment what a surprise!) qu'elles sont parfois employées de façon ironique ou dans le cadre d'une routine sociale où l'exclamative correspond à une sorte d'exclamation forcée pour répondre aux attentes de l'autre. Il faudra donc se demander si les deux exclamatives du corpus correspondent à une véritable fonction d'exclamation.

5 A nos yeux, c'est précisément l'absence de relation univoque entre une forme et une fonction qui justifie l'intérêt d'une approche dite "pragmatique", la pragmatique s'intéressant de façon générale à ce que l'on fait quand on parle (voir le titre How to do things with words choisi par J. L. Austin, 1962, l'un des fondateurs de la pragmatique moderne). Les questions théoriques que soulève une approche pragmatique de l'exclamation sont finalement les suivantes : quelle est exactement la «fonction » de l'exclamation? Que fait-on lorsqu'on s'exclame? Y a-t-il d'ailleurs une réponse homogène à cette question? La diversité des formes du corpus permet-elle de penser une unicité dans cette fonction d'exclamation?

6 Ces questions sont d'autant plus cruciales que, comme le notent Beijer (2002) et Merin \& Nikolaeva (2008), les fondateurs de la linguistique pragmatique (Austin 1962, Searle $1969,1975)$ ne se sont pas posé ces questions : l'exclamation ne constitue pas un objet d'étude dans leurs ouvrages. Dans les travaux pragmatiques ultérieurs, l'acte de langage qu'est l'exclamation semble encore difficile à cerner : il se rattache aux notions assez floues de l'expression et de l'expressivité (en référence à la fonction " expressive » du langage parmi les fonctions définies par R. Jakobson), en lien avec un état émotionnel. Aussi Sadock \& Zwicky (1985: 162) associent-ils l'exclamation ${ }^{6}$ à " a strong emotional reaction ", par opposition aux déclaratives, qui reposent sur une simple évaluation d'ordre intellectuel (« intellectual appraisal ») ${ }^{7}$. C'est donc aussi cette 
supposée composante émotionnelle de l'exclamation que le présent travail souhaite réexaminer.

7 La première partie sera consacrée à un essai de définition de l'approche pragmatique adoptée, dans une perspective épistémologique. Dans une deuxième partie, nous examinerons les formes d'exclamation du corpus en lien avec la notion d'acte de langage, centrale dans la pragmatique searlienne (J. Searle 1969) : si l'exclamation est bien un acte de langage, de quel acte s'agit-il exactement? Quelles sont ses caractéristiques? Enfin, dans une troisième et dernière partie, nous proposerons, en nous appuyant sur d'autres travaux en pragmatique plus centrés sur les émotions, d'envisager l'exclamation moins comme un acte lié à la subjectivité d'un locuteur que comme acte dirigé vers l'autre, dans une dimension intersubjective.

\section{Approche pragmatique : essai de définition}

\section{1. Pragmatique ou pragmatiques?}

8 Comme c'est le cas pour la plupart des approches ou théories linguistiques, l'approche pragmatique semble caractérisée par une certaine hétérogénéité, ce qui laisse à penser qu'il n'existe pas une et une seule approche pragmatique, mais bien des approches pragmatiques plurielles. Citons à titre d'illustration l'introduction à la collection Handbook of Pragmatics, consultée en ligne en octobre $2016^{8}$ :

This new landmark series of nine self-contained handbooks provides a comprehensive and up-todate overview of the entire field of pragmatics. It is based on a wide conception of pragmatics as the study of intentional human interaction in social and cultural contexts. The series reflects, appraises and structures a field that is exceptionally vast, unusually heterogeneous and still rapidly expanding. Indepth articles by leading experts from around the world discuss the foundations, major theories and most recent developments of pragmatics including philosophical, cognitive, sociocultural, contrastive and diachronic perspectives. " (nous soulignons).

Cette première définition met au jour l'hétérogénéité et l'interdisciplinarité constitutives du champ de la pragmatique. Malgré ce foisonnement dans les études se réclamant de la pragmatique, il parait important, d'une part, de circonscrire l'approche pragmatique que nous adoptons dans cet article à une approche proprement linguistique, laissant de côté les problématiques plus sémiotiques, sociologiques ou anthropologiques ; d'autre part, de rappeler brièvement, sur le plan épistémologique, comment s'est développé et constitué ce courant linguistique pragmatique, en le rattachant à quelques figures fondatrices.

\section{2. Les «pères fondateurs »}

Comme le rappellent Fuchs \& Le Goffic (1992: 129), « le courant pragmatique est, au moins au départ, d'inspiration logico-philosophique et s'inscrit dans une mouvance anglo-saxonne bien implantée aux Etats-Unis, en Grande-Bretagne et en Allemagne (et, à date plus récente, en France) ». Les premiers travaux se réclamant de cette mouvance sont ceux de Austin (1962), Searle $(1969,1975)$ et Grice (1975) ${ }^{9}$. Rappelons ici que ces ouvrages sont l'œuvre de philosophes du langage, et non pas de linguistes, qui s'intéressent à ce qu'ils appellent le «langage ordinaire». La pragmatique est donc d'abord philosophique avant d'être linguistique et «s'attache à décrire les conditions 
d'emploi du langage dans des situations effectives et à dénoncer les insuffisances d'une sémantique logique pour rendre compte du sens, de la référence et de la vérité " (Fuchs \& Le Goffic $1992: 135$ ). Cette théorie n'a pas été créée ex nihilo, mais, comme le note Blanchet (1996: 10-11), trouve son inspiration dans un retour à la rhétorique antique de Platon et Aristote, qui s'intéressaient déjà à la relation entre la signification des mots et la fonction du langage.

11 Les spécialistes d'histoire linguistique s'accordent à dire que c'est l'ouvrage de Austin, How to do things with words / Quand dire c'est faire, paru en 1962, qui constitue l'ouvrage fondateur de cette mouvance théorique. Cet ouvrage peut être considéré comme le résultat de la découverte que certains énoncés ne décrivent rien et ne sont pas analysables en termes de vérité et de fausseté, mais "agissent»: il s'agit de la découverte des énoncés appelés " performatifs ». Prenons comme exemple I declare the meeting open: dire cet énoncé suffit à marquer le début de la réunion, et, sans cette énonciation, la réunion ne peut débuter. Par le biais du performatif, c'est en fait un tournant épistémologique important qui se dessine : le performatif révèle que le propre du langage n'est pas lié à un élément interne à la langue appréhendable par une analyse en constituants (par exemple les morphèmes), mais l'acte d'énonciation en tant que tel - ce qu'Austin appelle « l'acte de langage » (« speech act » en anglais).

J. Searle $(1969,1975)$ développe ensuite la théorie des actes de langage, selon laquelle "proférer une énonciation, c'est accomplir un certain type d'acte, appelé «acte illocutionnaire $\mathrm{e}^{10} »(\mathrm{ex}$ : questionner, ordonner, promettre, suggérer, etc.) 》 (Fuchs \& Le Goffic 1992 : 137-138). Cette théorie met l'accent sur la distinction entre la signification d'un énoncé et la façon dont cet énoncé est utilisé en contexte. Plus précisément, elle repose sur la distinction de trois niveaux :

- le locutoire : ce qui est dit

- l'illocutoire : ce qui est « fait » par le langage

- le perlocutoire : l'effet produit, mais qui est contingent à la situation. Par exemple, l'acte de réconforter n'est pas lié à un énoncé particulier (contrairement à l'acte de baptiser, qui passe forcément par l'énoncé « je te baptise ») mais dépend largement de l'interprétation de l'interlocuteur.

13 Les actes de langage reposent en outre sur un certain nombre de règles, notamment des règles de sincérité («sincerity rules») : un acte ne fonctionne que si l'énonciation est sincère ${ }^{11}$. Ainsi, le performatif «I forgive you » ne fonctionne que si le locuteur accorde véritablement son pardon à l'autre. Nous reviendrons dans la troisième et dernière partie sur la pertinence de ce critère de sincérité, qui fait problème dans une approche intersubjective et sociale des émotions.

\section{3. Pragmatique et réorientation des problématiques linguistiques : un tournant épistémologique}

Les travaux de Austin et de Searle vont en fait permettre, sur le plan épistémologique, une réorientation des problématiques linguistiques : s'opère un recentrage sur le faire qui entraîne finalement un recentrage sur " celui qui fait ». Ce recentrage est bien mis en évidence dans les définitions proposées dans la littérature. Fuchs \& Le Goffic (1992 : 135) définissent ainsi la pragmatique comme l'« étude des signes en rapport avec leurs usagers" (nous soulignons). Pour Récanati (1981: 29), la pragmatique «s'occupe de l'utilisation des phrases » (nous soulignons). Jusqu'alors, la linguistique - dominée par 
les courants structuraliste d'une part, générativiste d'autre part - n'était pas centrée sur ces problématiques. De façon un peu schématique, on pourrait dire que la linguistique structuraliste héritée de Saussure consistait à étudier un système formel, interne à la langue (par opposition à la parole), sans prise en compte de «celui qui parle ", le locuteur. Quant à la grammaire générative développée par Chomsky, elle est fondée sur l'étude de la compétence par opposition à la performance - ce qui aboutit là aussi, à l'absence de prise en compte du locuteur. La pragmatique repose au contraire sur la croyance que l'étude de la signification ne peut passer que par une prise en compte du locuteur et de son action, par la parole, sur l'interlocuteur. La réorientation dont il est question peut donc être vue, ainsi que le note Blanchet (1996), comme une externalisation de l'objet d'étude de la linguistique et un dépassement de la sémantique, qui ne prend en compte que ce qui est interne à la langue. Prenons un exemple très simple: l'énoncé Changing tyres can be dangerous. Une approche sémantique analysera ce can comme exprimant une possibilité sporadique. Dans le cadre d'une analyse pragmatique, l'accent sera mis sur le rôle de ce can comme avertissement à l'autre : le sporadique est utilisé pour dire à l'autre de se méfier. On voit bien, par cette notion d'avertissement dans le cadre d'une relation intersubjective, que la pragmatique offre finalement une interface entre le linguistique et l'extralinguistique, ce que n'autorise pas la sémantique classique ${ }^{12}$. Mais l'on voit aussi que sémantique et pragmatique ne s'opposent pas : l'analyse pragmatique ne vient pas en remplacement de l'analyse sémantique, mais plutôt en complément de celle-ci.

Ce recentrage sur l'utilisation du langage par une instance subjective, en relation avec d'autres instances subjectives, va permettre, dans une certaine mesure, un rapprochement entre la pragmatique à l'anglo-saxonne que nous venons de présenter, et tout un courant linguistique européen : le courant énonciatif. De façon là encore très schématique $^{13}$, on peut considérer que ce courant commence avec les travaux de Guillaume et Bally, mais que c'est Benveniste (1974) qui est le premier théoricien de l'énonciation, définie par lui comme " la mise en fonctionnement de la langue par un acte individuel d'utilisation " (ibid.: 80). Plus récemment, ce sont surtout Culioli et Ducrot qui sont considérés comme les plus importants représentants de ce courant énonciatif.

On peut en fait dégager deux points communs à la pragmatique telle que nous l'avons définie plus haut et à l'énonciation. D'abord, les deux mouvances conçoivent le langage comme un acte émanant d'une instance subjective, susceptible d'agir sur autrui. Ensuite, elles mettent toutes deux en lumière l'importance du contexte : la signification d'une unité se construit en contexte (au sens linguistique de «co-texte»: environnement syntaxique, lexical etc. de l'énoncé, et également au sens de contexte extralinguistique). Il nous semble ainsi qu'il s'agit de deux approches tout à fait réconciliables, à deux conditions. Premièrement, à condition que l'on définisse précisément le périmètre de la pragmatique en termes linguistiques, ce qui implique d'exclure les approches communicationnelles qui étudient les conditions sociales, économiques, politiques de production des énoncés, parfois aux dépens de l'attention portée aux marqueurs linguistiques eux-mêmes. Cela revient finalement à veiller à ce que la réorientation épistémologique initiée par la pragmatique ne soit pas trop externalisante. Deuxièmement, à condition que l'on ne conçoive pas la pragmatique comme une théorie du langage purement «utilitariste». Une telle conception de la pragmatique serait effectivement irréconciliable avec l'énonciation : elle reviendrait à 
réduire le langage à un moyen de communication par lequel le locuteur « veut dire et faire » quelque chose de fixe, dans le cadre d'une vision intentionnaliste du langage qui postulerait une signification stabilisée. Or, bien sûr, le fonctionnement du langage est beaucoup plus complexe que ça : le «sens » est foncièrement dynamique, instable et se co-construit dans l'échange intersubjectif, dans la mesure où le sujet parlant ne sait pas nécessairement ce qu'il veut dire, et que ce qu'il dit n'a pas forcément comme but intentionnel d'agir sur autrui de façon précise. En outre, l'autre peut détourner et reconstruire ce qui a été dit - comme par exemple feindre de ne pas relever l'ironie de l'exclamative "what a convenient location!» de notre corpus (voir notre troisième et dernière partie).

17 Cette conception utilitariste et simpliste de la pragmatique, dont témoignent effectivement certains travaux ${ }^{14}$, explique la méfiance de certains énonciativistes envers cette approche, méfiance que l'on retrouve par exemple dans la réponse d'A. Culioli à une question de C. Normand concernant la position de la Théorie des Opérations Enonciatives face aux trois grands domaines de la linguistique (syntaxe / sémantique / pragmatique) :

" ça déborde toujours, la syntaxe déborde dans la sémantique, la sémantique dans ce qu'on a appelé la pragmatique, mais ce terme m'inquiète beaucoup : il y a ce que j'ai appelé la "pragmatique intégrée » - Ducrot a repris le terme - il y a la pragmatique à l'anglo-saxonne qui est nettement (culturellement) liée à une certaine conception utilitariste... » Culioli \& Normand (2005: 107).

18 Ainsi, pour nuancer le rapprochement que font Fuchs \& Le Goffic (1992) entre énonciation et pragmatique, nous parlerons plutôt d'une convergence possible entre énonciation et une certaine conception de la pragmatique, conception qui prend en compte la complexité de la co-construction du sens. Quelle peut alors être la différence entre l'approche pragmatique adoptée ici et une approche énonciative? L'approche énonciative nous semble plus englobante : syntaxe, sémantique et pragmatique ne sont pas séparées mais intégrées (d'où le terme de " pragmatique intégrée » mentionnée par Culioli dans sa citation- voir aussi Mélis 2012) dans un système global qui vise à reconstruire les opérations énonciatives abstraites qui sous-tendent l'emploi des marqueurs, dans l'optique de dégager une opération invariante pour chaque marqueur. Comme le note en outre Mélis (2012), le couple énonciateur-co-énonciateur sur lequel reposent les théories énonciatives se situe à un niveau d'abstraction absent de la pragmatique, qui s'intéresse de façon plus externe au couple locuteur-interlocuteur. Mélis souligne ainsi que la relation entre énonciateur et co-énonciateur est « distinct[e] de la relation pragmatique entre locuteur et allocutaire »(p.63), dans la mesure où l'énonciateur est plus qu'un simple «émetteur» et le co-énonciateur, qui est notamment pris en compte dans l'élaboration de l'énoncé, plus qu'un simple " récepteur ». Ce sont " des sujets pris dans des situations à la fois empiriques et en même temps liées à des représentations imaginaires du statut de chacun des sujets par rapport à l'autre » (Culioli 2002, cité par Mélis 2012 : 66).

19 Par conséquent, l'approche pragmatique que nous allons développer a sans doute un pouvoir d'abstraction moins fort et se concentre plus modestement sur la fonction ou les fonctions de l'exclamation en discours, dans le but de mettre en lumière ce qui se joue dans le rapport intersubjectif. Il ne s'agit pas non plus de défendre à tout prix un point de vue purement pragmatique de l'exclamation : l'enjeu de cet article est aussi de cerner les limites de la pragmatique, ou en tout cas d'une certaine pragmatique. 


\section{Exclamation et acte de langage : assertion et expression}

20 C'est d'abord en nous appuyant sur la notion d'acte de langage développée par Austin et Searle que nous allons analyser les occurrences du corpus. Sur le plan méthodologique, nous avons choisi d'opérer un va-et-vient entre ces occurrences et les outils théoriques utilisés dans la littérature sur l'exclamation dans un cadre pragmatique. Nous cherchons ainsi à voir dans quelle mesure ces occurrences confirment ou infirment les hypothèses théoriques défendues dans la littérature.

Un certain nombre de travaux essaient de circonscrire l'acte de langage à associer aux formes d'exclamation. Il s'agit là d'une question difficile car, comme le notent Merin \& Nikolaeva (2008) et Beijer (2002), il n'y a pas mention chez Searle d'un acte de langage spécifiquement " exclamatif ». Il y aurait peut-être ainsi une lacune originelle dans le modèle de Searle. Parmi les travaux visant à combler cette lacune, nous nous concentrerons principalement sur ceux de Michaelis (2001) et de Rett $(2008,2011)$. Le point commun à ces deux auteures est qu'elles postulent que la fonction d'exclamation, qui peut prendre plusieurs formes dans leur approche puisqu'elles ne se limitent pas à l'étude des exclamatives en what/how, met en jeu deux des actes de langage définis par Searle : l'acte assertif d'une part, l'acte expressif d'autre part.

Rappelons ici que Searle (1975 : 354-361) a défini dans sa théorie cinq actes de langage : les assertifs appelés aussi « représentatifs » (assertion, affirmation...) par lesquels les mots s'ajustent au monde pour le représenter; les directifs (ordre, demande, conseil...) par lesquels le monde doit s'ajuster aux mots; les promissifs (promesse, offre, invitation...), qui fonctionnent comme les directifs en termes d'ajustement entre le monde et les mots; les expressifs (félicitation, remerciement...), pour lesquels aucune direction d'ajustement n'est définissable; et enfin, les déclaratifs (déclaration de guerre, nomination, baptême...), qui témoignent d'une direction d'ajustement double (mots - monde / monde - mots). Nous nous concentrerons ici sur les assertifs et les expressifs. La différence majeure entre assertifs et expressifs est liée à la relation qu'ils établissent entre monde et mots. Dans l'acte assertif, les mots décrivent le monde et la proposition peut donc être jugée vraie (si les mots décrivent effectivement le monde) ou fausse (si ce n'est pas le cas). Dans l'acte expressif, aucune relation de ce type n'existe : cet acte exprime l'attitude du locuteur vis-à-vis d'un certain état de fait et la proposition ne peut donc être jugée vraie ou fausse.

L'exclamation serait donc un « acte de langage » complexe qui associerait deux actes de langage pourtant distincts dans la théorie de Searle (1975). On voit déjà que la pragmatique searlienne se heurte à l'étude de ce phénomène linguistique, qui constituerait un acte de langage particulier, unique en son genre, hybride (voir sur ce point Beijer 2002). Rett $(2008,2011)$ théorise plus avant le lien entre ces deux actes de langage au sein de l'exclamation : l'exclamation est pour elle d'abord un acte expressif mais qui implique (au sens de «entail» en anglais, c'est-à-dire implique nécessairement, quel que soit le contexte d'emploi) une assertion.

Maintenant que le caractère hybride de l'exclamation entre expression et assertion est posé, deux questions théoriques majeures émergent: d'une part, qu'asserte précisément l'exclamation? D'autre part, qu'exprime-t-elle vraiment? Nous allons d'abord tenter de répondre à la question concernant l'assertion. 


\subsection{Michaelis (2001) : contenu propositionnel et scalarité} soulignons). déclaratives :

Selon Michaelis (2001), la dimension assertive de l'exclamation ${ }^{15}$ associe deux caractéristiques : un contenu propositionnel présupposé et l'assertion d'un haut degré.

La première caractéristique est d'ordre sémantique et constitue un point commun entre les exclamations et les déclaratives :

"The major semantic feature which distinguishes exclamations from interjections is also the major semantic property that exclamations share with declaratives: recoverable propositional content. Both exclamations and declaratives linguistically encode a proposition which the speaker assumes to be true " (Michaelis, 2001: 1040, nous

Ainsi, les formes d'exclamations présupposent un contenu propositionnel du type sujet-prédicat, de façon explicite dans certains cas, implicite mais récupérable en contexte dans d'autres cas. Cette définition permet de rendre compte :

- de certaines exclamations prenant la forme de déclaratives («sentence exclamations »), mais pas de toutes (voir plus bas)

- des exclamatives en what et how, qu'elles soient réduites, c'est-à-dire averbales, ou non (voir l'exclamative «what a convenient location!", pour laquelle on peut aisément rétablir la copule de la prédication : what a convenient location this is !)

- d'autres énoncés averbaux, sans morphosyntaxe exclamative, comme 1. 10 «Stella, oh, Stella, Stella! Stella for Star ! ». Il y a bien là aussi un contenu propositionnel sous-jacent ${ }^{16}$, que l'on peut faire apparaître en glosant par : Stella stands for star ou Stella is a star.

Néanmoins, cette conception du phénomène conduit à exclure de l'exclamation les interjections seules : oh ou ah n'ont pas de contenu propositionnel. On pourra noter à ce propos que le corpus ne contient pas de soulignage d'interjections seules. Elles ne sont soulignées qu'en co-occurrence avec des formes ayant un contenu propositionnel. Or, si l'on raisonne en termes de "fonction d'exclamation ", alors certaines interjections ont bien leur place au sein de l'exclamation (voir Merin \& Nikolaeva 2008, qui proposent d'inclure certaines interjections dans l'acte d'exclamation). Paradoxalement, Michaelis elle-même souligne une parenté de fonctionnement entre certaines interjections et les exclamatives : «some [interjections], like Hey! or Oh My God! have a function like that of exclamatives » (Michaelis 2001 : 1039), ce qui montre bien la difficulté qu'il y a à restreindre l'exclamation à la présupposition d'un contenu propositionnel.

Michaelis essaie de montrer en outre que l'exclamation asserte un haut degré. Cette deuxième caractéristique permet, cette fois-ci, de distinguer formes d'exclamation et

"Exclamations, unlike declaratives, presuppose that the proposition expressed is mutually known by speaker and hearer. The presupposed proposition is one which involves a scalar degree. The degree itself is not mutually presupposed » (Michaelis 2001: 1040)

Cette propriété explique la présence fréquente de marqueurs anaphoriques, tel l'adverbe so, que l'on retrouve effectivement 1. 131 «It's so becoming to you!", qui présuppose it's becoming to you to $x$ degree, $\mathrm{x}$ étant un degré élevé. La notion de présupposition permet, toujours selon Michaelis, de fonder un rapprochement entre exclamation et question (ouverte) sur le plan pragmatique: «[exclamations and questions] have the same pragmatic starting point: the speaker takes for granted, and presumes that the hearer is willing to take for granted, the proposition [presupposed] » 
(ibid.: 1047). Cette définition implique donc que l'exclamation n'est jamais de l'ordre de l'information nouvelle.

31 L'assertion d'un haut degré conduit ainsi à ne retenir comme formes d'exclamation que celles impliquant un jugement scalaire, c'est-à-dire :

- les exclamatives (structures en what et how)

- les exclamations de phrase contenant un adverbe de degré explicite, comme l'énoncé

1. 131 «It's so becoming to you!»

- les formes pour lesquelles il est possible de reconstruire un jugement scalaire implicite. On peut par exemple gloser 1. 66 "Why, that you had to live in these conditions! »

${ }^{17}$ par it's so unfair that you had to live in these conditions!

Se trouvent en revanche exclues les exclamations de phrase du type « Yes, types is right! ", au motif qu'elles ne présupposent aucune proposition contenant un jugement scalaire.

Le critère de la présupposition, qui rejoint celui de la factivité développée par exemple par Ginzburg \& Sag $(2000)^{18}$, fait en fait problème, et ce pour deux raisons. D'abord, comme nous venons de le voir, parce qu'il conduit à limiter l'exclamation aux énoncés liés au haut degré, mais aussi, comme le notent plusieurs auteurs (Rett $2011: 435$, Heine et al., communication personnelle à propos de travaux en cours), parce qu'il exclut les "news-reporting utterances ", c'est-à-dire les exclamations introduisant une information nouvelle, ne reposant pas sur un savoir partagé (du type: Look! It's snowing!).

33 Au terme de cette discussion, il apparait que la position défendue par Michaelis (2001) témoigne d'une définition trop stricte de l'exclamation, qui conduit à exclure du phénomène de nombreuses formes d'exclamation présentes dans le corpus: les interjections, qui n'ont pas de contenu propositionnel, les formes qui ne reposent pas sur un jugement scalaire et les formes qui ne sont pas présupposantes. Faut-il pour autant dénier toute pertinence à l'acte assertif pour rendre compte de l'exclamation ? Pour le cas particulier des interjections, il semble que oui : ces formes relèvent de l'expression et non de l'assertion. Pour les autres formes, il y a bien contenu propositionnel, mais sans qu'il y ait nécessairement jugement scalaire - c'est en tout cas la position défendue par Rett (2011) et que nous reprenons ici à notre compte.

\section{2. Expression}

34 A ce stade de l'analyse, on comprend que dans le modèle pragmatique présenté, s'exclamer consiste à asserter (du moins si l'on exclut les interjections), mais pas seulement : s'ajoute à l'assertion un " plus » qui permet de distinguer ce qui relève de l'exclamation de ce qui relève des énoncés purement déclaratifs : un acte expressif. Comme nous l'avons rappelé précédemment, l'acte expressif défini par Searle (1975: 356-358) concerne les énoncés par lesquels le locuteur exprime son attitude. Cet acte repose en outre sur un postulat important: en vertu de la règle de sincérité, l'expression doit refléter l'état émotionnel du locuteur, sinon l'acte échoue.

Or, comme le note Beijer (2002), les actes expressifs mentionnés par Searle se réduisent à des énoncés performatifs certes liés à l'attitude du locuteur mais dans un contexte social - tels les actes de remercier ou de s'excuser. A ainsi été laissé de côté par Searle tout un ensemble de marqueurs, que Beijer (2002) nomme "expressive/emotional utterances", qui ne fonctionnent pas comme les performatifs. En effet, quelles que 
soient leurs formes, les exclamations du corpus ne «font » pas l'événement auxquelles elles réfèrent à la manière des performatifs.

Pour rendre compte de la spécificité de l'exclamation au sein des actes expressifs, c'est la notion d'expression émotionnelle qui est invoquée dans la littérature, notamment en relation avec la catégorie psychologique de la surprise.

\subsubsection{Expression émotionnelle : exclamation et surprise}

Plusieurs travaux pragmatiques sur l'exclamation développent ainsi l'hypothèse que l'exclamation repose sur un acte expressif de nature émotionnelle en lien avec un ressenti de surprise. Voici trois citations représentatives de cette hypothèse :

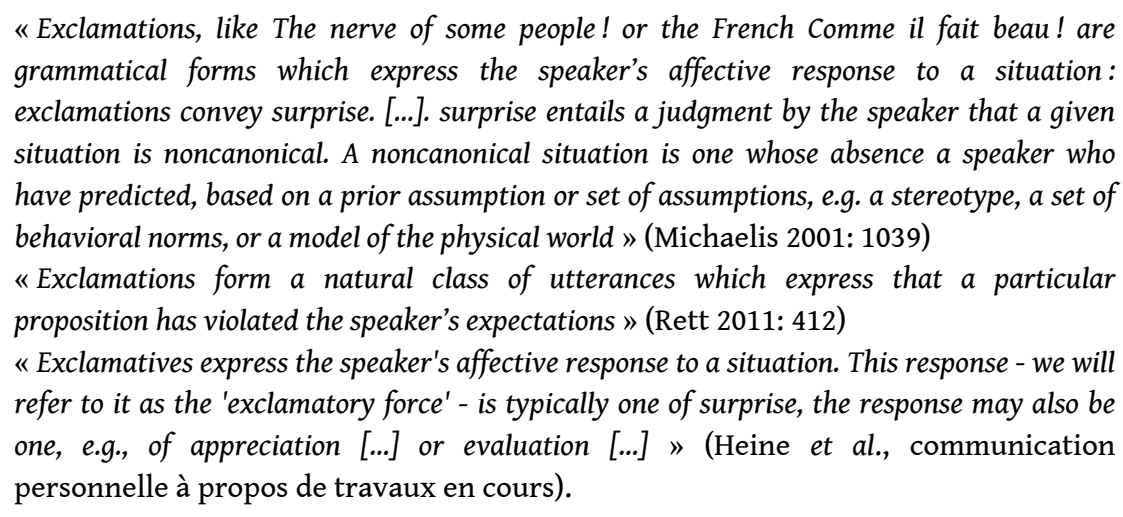

Malgré certaines différences - on voit notamment que le lien entre exclamation et surprise est moins nettement affirmé chez Heine et al. ${ }^{19}$ - ces trois citations témoignent d'un double consensus concernant la fonction d'exclamation :

- l'exclamation est vue, prototypiquement, comme l'expression d'un décalage ou d'une discordance sur le plan cognitif entre un état de fait et des représentations initiales (une sorte d'horizon d'attente), ou entre un nouvel état de fait et l'état de fait précédent, ce qui se traduit sur le plan émotionnel par un ressenti de surprise ${ }^{20}$.

- le choix de la surprise comme fonction majeure ou en tout cas prototypique de l'exclamation permet de souligner l'absence de valence $^{21}$ dans l'exclamation: la discordance peut être aussi bien positive (comme dans «It's so becoming to you!») que négative (voir «Why, that you had to live in these conditions!») selon l'environnement contextuel (lexique, négation, situation de locution etc.).

\subsubsection{Expression et indexicalité}

Autre caractéristique essentielle de l'acte expressif qu'est l'exclamation : sa dimension indexicale, déjà notée par Michaelis (2001:1039-40), qui, rappelons-le, ne distingue pas " exclamation » d' " exclamative »: " Exclamatives have the indexical function of expressing speaker perspective». Cela se traduit sur le plan des marqueurs par une affinité entre exclamation et déictiques, affinité que l'on retrouve dans notre corpus par exemple dans l'exclamation « Why, that you had to live in these conditions! ».

Dans un travail plus récent, Rett \& Murray (2013: 455) tentent de formaliser cette dimension, en dégageant quatre critères permettant de définir l'expression de la discordance par l'exclamation (par opposition à sa simple assertion dans des énoncés du type I'm surprised). A propos de l'exclamation de phrase wow, John arrived on time!, elles affirment que : 
1. L'expression de la surprise ne peut être niée

\# No, you are not surprised n'est pas un énoncé équivalent. Seul le contenu peut être nié : No, John didn't arrive on time.

2. On ne peut pas enchâsser l'exclamation de phrase sous une négation ou un discours direct :

John didn't arrive on time! ne peut pas exprimer l'absence de surprise du locuteur face à la ponctualité de John. De la même façon, dans le cadre du discours indirect, Mary said John arrived on time! exprime forcément la surprise du locuteur face à l'affirmation de Marie, et non face à la ponctualité de John.

3. L'expression de la surprise est toujours orientée vers le locuteur : la surprise ne peut être que celle du locuteur et l'intonation est celle de l'exclamation, et non de l'interrogation.

4. L'exclamation est contrainte par la « restriction de récence " (« recency restriction »): le locuteur ne peut s'exclamer longtemps après l'événement ou le discours jugé surprenant.

Ces quatre critères formels forgent une distinction fondamentale, dans la lignée de Potts (2005), entre simple assertion de surprise et l'exclamation de surprise (qui associe assertion et expression). L'assertion simple, comme dans I am surprised/je suis surpris(e), peut faire l'objet d'une remise en cause dans l'interaction verbale : no, you're not, I can see you're angry ${ }^{22}$. A l'inverse, l'expression de la surprise via l'exclamation est indexicale et inaccessible à l'interlocuteur, qui ne peut remettre en cause que le contenu.

On voit ici que la dimension expressive de l'exclamation fait l'objet d'un fort consensus dans les travaux de la mouvance pragmatique: l'ensemble des auteurs cités font l'hypothèse que l'exclamation exprime un décalage cognitif, entre un état de fait et des représentations initiales, ou un nouvel état de fait et l'état précédent, décalage qui donne lieu à une émotion de surprise.

Cette position nous paraît pertinente par certains aspects, puisqu'elle permet de rendre compte de nombreuses occurrences du corpus. Nous souhaitons plus particulièrement postuler qu'il existe un continuum de formes d'exclamation exprimant ce décalage ${ }^{23}$. A une extrémité du continuum, on trouve ainsi des énoncés marqués par une disfluence discursive : des énoncés averbaux ou des exclamations de phrase ne contenant pas de lexèmes évaluatifs, associées à des interjections et/ou des répétitions marquant une perte de contrôle du locuteur. Citons à titre d'exemple les énoncés averbaux «Only Poe! Only Mr. Edgar Allan Poe!» et "Stella, oh, Stella, Stella! Stella for Star!», caractérisés tous deux par des répétitions ${ }^{24}$. On retrouve bien dans les deux cas un décalage entre deux états de fait : le premier énoncé est lié à la soudaine révélation par la locutrice Blanche que seul Poe, et non pas elle, pourrait décrire les lieux; le second est déclenché par l'arrivée de Stella, qui entraîne chez Blanche une nouvelle perception de la «luminosité » de sa sœur, qui porte si bien son prénom. Dans les deux cas, la discordance prend ainsi la forme d'une sorte d'auto-révélation ou d'épiphanie pour Blanche, dont les émotions sont au cœur du corpus à l'étude. En plus de la répétition et des interjections (voir "Oh» dans le dernier énoncé cité), le corpus contient un marqueur de disfluence syntaxique: la subordonnée en that introduite par l'interjection why, "Why, that you had to live in these conditions!». Cette subordonnée n'est rattachée à aucune principale, et constitue à ce titre selon Evans (2007 : 403-405) un cas d'insubordination. Cette forme exprime bien elle aussi un décalage sur le plan 
cognitif, décalage ici entre la réalité de la situation observée (voir le déictique these) et ce qui était attendu sur le plan des représentations - à savoir un logement décent.

A l'autre extrémité du continuum se trouvent des formes d'exclamation témoignant d'une plus forte rationalisation de ce décalage cognitif. Cette rationalisation prend la forme d'un jugement modal marqué lexicalement, sans disfluence syntaxique, comme dans les exclamations de phrase ${ }^{25}$ « Yes, types is right! », «It's so becoming to you! » ou " It's not that bad at all!». Les adjectifs right, becoming et bad marquent une évaluation ou une appréciation (avec valence positive pour les deux premiers, négative pour le troisième) qui témoigne d'une certaine prise de distance de la locutrice face à l'état de fait discordant : l'évaluation et l'appréciation impliquent que la surprise soit déjà en partie intégrée sur le plan cognitif. Dans le premier énoncé, la locutrice manifeste une fois de plus une sorte d'épiphanie face à la justesse du nom "types " pour décrire la réalité. Dans les deux autres énoncés, l'exclamation naît d'un décalage observé dans l'extralinguistique entre la situation et les représentations de la locutrice.

51 Ces deux pôles du continuum peuvent être vus comme des pondérations différentes de l'expression et de l'assertion. Les formes plus spontanées, moins articulées, sont plus du côté de l'expression, parfois même sans aucun contenu propositionnel si l'on inclut les interjections. Les formes plus élaborées verbalement et contenant des lexèmes évaluatifs ou appréciatifs relèvent plus nettement de l'assertion : par l'assertion d'un contenu propositionnel de type évaluatif ou appréciatif, ces énoncés correspondent à une phase de plus forte intégration cognitive de la surprise. Prototypiquement, cette intégration prend la forme d'une évaluation ou appréciation du décalage perçu. Dans un autre énoncé du corpus, elle ne passe cependant pas par une évaluation/ appréciation, mais par une incitation à l'action : ainsi, l'impératif «Turn that over-light off!» associe perception d'un décalage (lumière désagréable) et acte directif.

Cette hypothèse d'un continuum de formes d'exclamation allant du moins intégré au plus intégré cognitivement pourrait être validée sur un plus grand corpus ${ }^{26}$, mais l'avantage de cette approche en termes de décalage cognitif est qu'elle permet de rendre compte de toutes les formes relevées dans le corpus, sans exclusion des interjections ou d'exclamations de phrase ne satisfaisant pas à la présupposition scalaire. Elle se heurte néanmoins à un problème majeur: si elle permet a priori d'intégrer toutes les formes possibles d'exclamation, est-elle vraiment pertinente pour analyser tous les énoncés? Il nous semble crucial de bien distinguer les formes en tant que telles, qui existent hors contexte, des énoncés, qui constituent une utilisation particulière d'une forme dans un contexte donné. Ainsi, en contexte, il nous semble difficile de voir dans les énoncés suivants : «What a convenient location!» et «It's just incredible, Blanche, how well you're looking!», l'expression d'une véritable émotion de surprise liée à un décalage entre un état de fait et des représentations. Il y a bien exclamation, mais en lien avec diverses stratégies intersubjectives, dirigées vers l'autre, soit à des fins ironiques, soit dans le cadre de conventions sociales.

53 Nous souhaitons ainsi montrer, dans la troisième et dernière partie, que la pragmatique héritée de Searle échoue à rendre compte de certains énoncés relevant de l'exclamation. C'est vers un autre type de travaux pragmatiques qu'il faut se tourner, travaux qui reposent sur une conception intersubjective, et plus largement sociale, des émotions, dans laquelle le linguistique n'est pas un décalque de l'émotionnel. 


\section{Exclamation et intersubjectivité : de l'expression à l'expressivité}

Dans le cadre pragmatique hérité de Searle, les deux énoncés qui nous posent ici problème seraient analysés comme "actes mensongers" («insincere speech acts»), c'est-à-dire comme des échecs de l'acte expressif. En effet, comme nous l'avons précisé lors de notre présentation de la théorie des actes de langage, un acte expressif ne fonctionne que si la règle de sincérité est respectée, c'est-à-dire si et seulement si l'acte correspond à l'état psychologique interne du locuteur. Or, dans le contexte donné, on comprend que la forme exclamative "What a convenient location!» est en fait à interpréter non pas comme this location is so convenient! mais comme this is not a convenient location at all! Dans le cas de l'exclamation de phrase dans laquelle est enchâssée une exclamative indirecte (extraposée) «It's incredible, Blanche, how well you're looking!», le contexte-avant suggère que la locutrice n'est pas vraiment sincère: on pourrait parler d'exclamation forcée, prononcée en réponse à une attente de Stella, désespérément en quête de compliments. S'agit-il pour autant d'actes mensongers, le terme «mensonge» reposant sur une intention délibérée de travestir la vérité ? Comme nous l'avons montré à propos d'autres exclamatives (voir Celle \& Lansari 2015), une analyse en termes de mensonge ne permet pas de comprendre le rôle discursif et interactionnel de l'exclamation, aussi peu sincère soit-elle : pourquoi le locuteur a-t-il malgré tout recours à une structure que l'autre va, ou en tout cas peut, identifier comme acte expressif d'exclamation?

Dans la pragmatique héritée de Searle, l'exclamation est d'abord vue comme l'expression d'un état interne du locuteur, c'est-à-dire comme un acte éminemment subjectif. Par conséquent, le rapport intersubjectif construit par l'exclamation reste peu et mal étudié dans ce type de travaux. Par exemple, Michaelis (2001:1040) affirme rapidement que le rôle de l'exclamation est informatif: "the speaker's purpose in exclaiming is to inform the hearer that the degree in question is extreme " (nous soulignons). Or, ce rôle informatif n'est pas très convaincant tant la notion factuelle d'information apparaît en contradiction avec la dimension émotionnelle de l'exclamation. Plusieurs travaux plus récents cherchent à combler cette lacune du modèle pragmatique et proposent d'opérer un changement de paradigme dans l'étude de l'exclamation, de la subjectivité à l'intersubjectivitée ${ }^{27}$. Les effets produits par l'exclamation sur l'interlocuteur et les réactions de ce dernier se trouvent donc désormais au centre des études sur ce phénomène linguistique.

Pour opérer ce changement de paradigme, nous nous appuyons pour notre part sur un certain nombre de travaux pragmatiques qui diffèrent de la mouvance searlienne et prennent en compte la dimension sociale de la manifestation linguistique des émotions $^{28}$. Ces travaux reposent sur l'idée-force qu'il ne faut pas confondre manifestation linguistique des émotions et vécu psychologique interne : le discours ne saurait se réduire à une extériorisation publique d'une émotion vécue intérieurement et il n'existe pas de relation miroir entre ressenti émotionnel interne et manifestation linguistique externe. Comme l'a bien montré Plantin (2011 : 143), les locuteurs peuvent ressentir de la surprise sans la manifester, dans une stratégie de contrôle; inversement, ils peuvent " afficher » (terme emprunté à Plantin 2011) une émotion sans véritablement la ressentir. Cette dissociation entre manifestation et ressenti n'est en fait pas nouvelle sur le plan épistémologique : Caffi \& Janney $(1994)^{29}$ montrent qu'elle 
est déjà en germe dans certains travaux du début du $20^{\mathrm{ème}}$ siècle, notamment dans ceux du philosophe Marty (1908) et du linguiste Bally (1926). A partir de ces travaux, qui postulent de distinguer deux types de communication (" mode vécu » et «mode pur » pour Bally, «communication émotive " et «communication émotionnelle " pour Marty), Caffi \& Janney (1994) redéfinissent deux modes de communication en lien avec les émotions : la communication «émotionnelle " et la communication " émotive ». La communication émotionnelle s'apparente à une manifestation spontanée des émotions (" a type of spontaneous, unintentional leakage or bursting out of emotion in speech », Caffi \& Janney 1994 : 328). Par contraste, la communication dite " émotive » met en jeu un contrôle du locuteur sur son discours, dans le cadre de diverses stratégies intersubjectives: "it has no automatic or necessary relation to "real" inner affective states. Rather, it is related to self-presentation and it is inherently strategic, persuasive, interactional and other-directed by its very nature ». (ibid.).

57 A la lecture de cet article, on comprend que les formes d'exclamation que nous avons examinées dans notre deuxième partie relèvent de la communication émotionnelle (mais à des degrés divers, en fonction de leur place dans le continuum cognitif allant du moins intégré au plus intégré) : ces formes sont l'expression d'un véritable décalage entre un état de fait et des représentations, décalage qui donne lieu à un vrai ressenti de surprise. En revanche, les énoncés «What a convenient location!» et «It's incredible, Blanche, how well you're looking!» correspondent au second mode de communication défini, où l'exclamation est mise au service d'une stratégie intersubjective.

58 Cette stratégie ne nous semble cependant pas du même ordre pour les deux énoncés. Pour l'énoncé "It's incredible, Blanche, how well you're looking!», la stratégie est liée à des règles sociales. Le contexte-avant montre bien que la locutrice ne fait que se conformer à l'attente de l'autre. Comme l'affirment Heath et al. (2012) dans leur étude multimodale sur la surprise en interaction, il ne s'agit pas tant d'un acte mensonger que d'une routine sociale, inconsciente car intériorisée,: "This is not to say that the emotion is disingenuous but rather that in this case, surprise is a sequentially and situationally appropriate response, not simply to the event, but to the ways in which the event is rendered visible and the obligations that arise and rest therein. " (ibid.: 216). La situation impose à la locutrice de manifester publiquement une émotion qu'elle ne ressent pas véritablement. Or cette dimension sociale de l'exclamation a largement été ignorée par la pragmatique ${ }^{30}$.

L'exclamative "What a convenient location!» repose sur une stratégie différente. Elle semble relever de l'emploi ironique des exclamatives, emploi bien répertorié dans les travaux en pragmatique (voir Potts 2005, Rett 2008, 2011) et analysé en termes de mensonge. Comme pour le cas précédent, nous récusons une telle analyse: l'ironie correspond pour nous à une mise en scène dans le cadre d'un positionnement intersubjectif particulier. Certes, il n'y a plus d'acte expressif au sens défini par Searle, mais un autre type d'expressivité est en jeu: l'expressivité «éthique " définie par Legallois \& François (2012 : 210-211) comme "promotion de soi ", par laquelle le locuteur met en scène ou dramatise son discours, ce qui est susceptible d'avoir plusieurs effets sur l'autre. Dans le cas de "What a convenient location!», la stratégie expressive est très complexe. L'énoncé se présente sur un plan fictif et correspond à ce que la locutrice voulait dire mais n'a pas initialement dit. Le fait qu'elle réintroduise dans le discours cette formulation semble a priori montrer qu'elle cherche finalement à ménager l'autre (après l'avoir culpabilisée frontalement avec « horrible place »). Mais la 
reformulation par "What a convenient location» est ironique: aucune des deux locutrices n'est dupe. Ainsi, la culpabilisation reste palpable: quels que soient ses efforts de reformulation, la locutrice ne peut s'empêcher de se faire plaindre.

$60 \mathrm{Au}$ terme de cette réflexion, on peut se demander si les "vraies" exclamations analysées dans notre deuxième partie ne relèvent pas elles aussi, du moins dans une certaine mesure, de cette même expressivité éthique: la propension des deux locutrices à s'exclamer dès qu'elles perçoivent un décalage cognitif correspond aussi à une posture particulière vis-à-vis de l'autre.

\section{Conclusion}

61 Adopter un point de vue pragmatique sur l'exclamation oblige à redéfinir aussi bien l'approche pragmatique que l'exclamation. Nous avons montré que la mouvance pragmatique est foncièrement hétérogène et se nourrit autant des travaux pionniers d'Austin et Searle sur les actes de langage que de travaux européens plus anciens et récemment redécouverts sur les émotions. Nous nous sommes efforcée de mettre en lumière les apports mais aussi les limites de l'héritage searlien, qui voit l'exclamation comme acte de langage associant assertion et expression d'une émotion de surprise qui doit être nécessairement ressentie par le locuteur. D'autres travaux s'intéressant à ce que l'on fait avec le langage montrent que la manifestation linguistique des émotions (par l'exclamation ou d'autres formes, d'ailleurs) ne se réduit pas à un décalque de l'état psychologique du locuteur, mais qu'elle peut servir diverses stratégies intersubjectives.

$\mathrm{Vu}$ sous l'angle triple de l'assertion, de l'expression et de l'expressivité, l'exclamation apparaît ainsi éminemment complexe et polyvalente tant dans sa forme que dans sa fonction : elle exprime prototypiquement un décalage cognitif entre un état de fait et des représentations, ou entre deux états de fait, mais elle peut également être motivée par des conventions sociales indépendantes d'un affect individuel, ou participer d'un positionnement particulier d'un locuteur vis-à-vis de son interlocuteur.

\section{BIBLIOGRAPHIE}

Ariel, M. (2010). Defining pragmatics. Cambridge: Cambridge University Press.

Austin, J. L. (1962). How to do things with words. Oxford : Oxford University Press. Trad. française

(1970), Quand dire, c'est faire. Paris : Seuil.

Bally, C. (1926). Le Langage et La Vie. Paris : Payot.

Beeching, K. (2016). Pragmatic Markers in British English : Meaning in Social Interaction.

Cambridge: Cambridge University Press. 
Beijer, F. (2002). The Syntax and Pragmatics of Exclamations and other Expressive/Emotional Utterances, The department of English in Lund: working papers in linguistics. https://lucris.lub.lu.se/ws/ files/5340344/624533.pdf Benveniste, E. (1974). Problèmes de linguistique générale, tome 2. Paris : Gallimard. Blanchet, P. (1996). La pragmatique. D’Austin à Goffman. Paris : Bertrand-Lacoste.

Caffi, C., Janney, R. W. (1994). « Toward a Pragmatics of Emotive Communication ». Journal of Pragmatics 22 (3-4) : 325-73.

Celle, A. (sous presse). « Surprise in Storytelling ». R. Nita \& F. Valetopoulos (éds.). Les sentiments à travers les corpus. Presses Universitaires de Rennes.

Celle, A. (à paraître). « Questions as indirect speech acts ». A. Celle \& N. Depraz (eds) Surprise at the Intersection of Phenomenology and Linguistics. [Consciousness and Emotion Series]. Amsterdam: John Benjamins.

Celle, A., Jugnet, A., Lansari, L. \& L'Hôte E. (2017). « Expressing and Describing surprise ». A. Celle \& L. Lansari (eds). Expressing and Describing Surprise. Amsterdam: John Benjamins. 215-244.

Celle, A., Jugnet, A., Lansari, L. \& Peterson, T. (à paraître). « Interrogatives in Surprise Contexts in English ». A. Celle \& N. Depraz (eds). Surprise at the Intersection of Phenomenology and Linguistics. [Consciousness and Emotion Series]. Amsterdam: John Benjamins.

Celle, A. \& Lansari, L. (2014). « 'Are you surprised?' / 'I'm not surprised'. Surprise as an argumentation tool in verbal interaction ». P. Blumenthal, I. Novakova \& D. Siepmann (eds.), Les émotions dans le discours. Emotions in discourse. Bern, New York: Peter Lang. 267-280.

Celle, A.\& Lansari, L. (2015). « Surprise et exclamation en anglais ». N. Depraz \& C. Serban (eds). La surprise à l'épreuve des langues. Paris : Hermann. 79-99.

Culioli, A. \& Normand, C. (2005). Onze rencontres sur le langage et les langues. Paris: Ophrys.

Evans, N. (2007). « Insubordination and its uses ». I. Nikolaeva (ed.), Finiteness: Theoretical and Empirical Foundations. Oxford : Oxford University Press. 366-431.

Faure, R. (2016). « Exclamations as multi-dimensional intersubjective items ». Revue de Sémantique et Pragmatique. Presses de l'Université d'Orléans. 40 : 7-40.

Fuchs, C. \& Le Goffic, P. (1992). Les linguistiques contemporaines. Repères théoriques. Paris : Hachette Supérieur.

Ginzburg, J., Sag, I., 2000, Interrogative Investigations : the Form, Meaning, and Use of English Interrogatives. Stanford : CSLI Publications.

Grice, H., 1975. « Logic and Conversation ». Syntax and Semantics 3: Speech Acts. New York: Academic Press. 41-58.

Guillaume, G. (1964). Langage et science du langage. Québec : Presses de l'Université de Laval, et Paris : Nizet.

Heath C., vom Lehn D., C. Jason \& Luff P. (2012). « Revealing Surprise, the Local Ecology and the Transposition of Action ». Perakyla A. et Sorjonen M.-L. (dir.), Emotion in Interaction. Oxford: Oxford University Press.

Heine, B., Kaltenböck G. \& Kuteva, T. (communication personnelle à propos de travaux en cours), «Exclamatives as a thetical category». 
Johansson, M. \& Suomela-Salmi, E. (2008). «Énonciation: French pragmatic approach(es) ». Handbook of Pragmatics online (https://benjamins.com/online/hop2/articles/eno1). Volume 12, $1-38$.

Lefeuvre, F. (1999). « Les 'marqueurs de prédication' dans la phrase averbale en français ». Verbum XXI, 4. 429-438.

Legallois, D. \& François, J. 2012. «Définition et illustration de la notion d'expressivité en linguistique ». N. Le Querler, F. Neveu, E. Roussel (eds). Relations, Connexions, Dépendances : Hommage Au Professeur Claude Guimier. Rennes : Presses universitaires de Rennes. 197-221.

Marty, A. (1908). Untersuchungen zur Grundlegung der allgemeinen Grammatik und Sprachphilosophie, Halle a. Salle : Niemeyer.

Mélis, G. (2012). « Les enjeux de la notion d'ajustement : pour une pragmatique intégrée radicale ». C. Filippi-Deswelle (ed). L'ajustement dans la TOE d'Antoine Culioli. 63-80.

Merin, A. \& Nikolaeva, I. (2008). Exclamative as a universal speech act category: a case study in decisiontheoretic semantics and typological implications. Ms., University of Konstanz and SOAS London University.

Michaelis, L. 2001. « Exclamative Constructions ». M. Haspelmath (ed). Language Typology and Language Universals: An International Handbook. Berlin, New York : Walter de Gruyter. 1038-50.

Micheli, R. (2014). Les émotions dans les discours. Modèle d'analyse, perspectives empiriques. Louvain-la-Neuve : De Boeck, Duculot.

Peräkylä, A. \& Sorjonen, M.-L. (éds) (2012). Emotion in Interaction. New York: Oxford University Press.

Plantin, C. (2011). Les Bonnes Raisons Des Émotions : Principes et Méthode Pour L'étude Du Discours Émotionné. vol. 94. Sciences Pour La Communication. Bern, New York: Peter Lang.

Potts, C. (2005). The Logic of Conventional Implicatures. Oxford: Oxford University Press.

Quirk, R., Greenbaum, S., Leech, G. \& Svartvik, J. (1985). A Comprehensive Grammar of the English Language. Cambridge : Cambridge University Press.

Récanati, F. (1981). Les énoncés performatifs. Paris : Les Éditions de Minuit.

Rett, J. (2008). « A Degree Account of Exclamatives ». Proceedings of SALT, 18. 601-618. <http:// elanguage.net/journals/salt/article/ download/18.601/1921>.

Rett, J. (2011). « Exclamatives, Degrees and Speech Acts ». Linguistics and Philosophy 34 (5), 411-442.

Rett, J. \& Murray, S. (2013). « A Semantic Account of Mirative Evidentials ». Proceedings of SALT, 23:453-72, http://elanguage.net/journals/salt/article/view/23.453/0. (consulté en 2014)

Sadock, J. \& Zwicky, A. (1985). « Speech Acts Distinctions in Syntax ». In Shopen, Timothy (ed.), Language Typology and Syntactic Description; 155-196. Cambridge: Cambridge University Press.

Schiffrin, D. (1987). Discourse Markers. Cambridge: Cambridge University Press.

Searle, J. (1969). Speech Acts: An Essay in the Philosophy of Language. London: Cambridge University Press.

Searle, J. (1975). « A taxonomy of illocutionary acts ». Language, Mind and Knowledge, Minnesota Studies in the Philosophy of Science. 344-369.

Tamba, I. (2007). La sémantique. Que Sais-Je ? Paris : Presses Universitaires de France. 